AUTHORS' REPLY We thank Dr Addy for his comments on our paper. There is an element of error in the predictions of the numbers of patients with cystic fibrosis, which relates to the estimates of the number of births and the statistical errors for each year extrapolated to the year 2000 . The assumptions of birth rate over the next decade are conservative and so may result in an underestimate of total numbers over the next 10 years. Our predicted numbers, derived from the death certificate data and the logistic model, compare well with those of the British Paediatric Society's survey of cystic fibrosis patients in 1986. This suggests that the model we have used is robust and further predictions will be reasonably accurate.

There is no evidence from the 1959-86 data that the improvements in survival are plateauing and so the assumption of continuing improvements is justified. Gene therapy and other new treatments are unlikely to be available for at least five more years and so will have little, if any, effect on survival Although there may be some imprecision in our estimates we can be very confident that children born over the next decade will have a median survival better than 30 years. It is therefore appropriate to use the conservatively projected figures from our predictive model rather than current figures, which will be unduly pessimistic, when we are counselling parents or prospective parents of children with cystic fibrosis.

The predicted improvements in survival do have implications for population screening. The identification of carriers (heterozygotes) and consequent prenatal screening may be difficult to justify as parents may not feel that termination is appropriate for a fetus with a potential median survival of 40 years In contrast, our data do not have any implications for neonatal screening, which may contribute to improvements in survival by identifying children with cystic fibrosis early in life.

STUART ELBORN D J SHALE Section of Respiratory Medicine, University of Wales College of Medicine, Llandough Hospital, Penarth, S Glamorgan CF6 1XX
J R BRITTON
Respiratory Medicine Unit, Respiratory Medicine Unit, City Hospital, Nottingham NP5 IPB

Invasive aspergillosis in a patient with idiopathic thrombocytopenic purpura without underlying lung disease

Dr L B Palmer and others (January 1991;46: 15-20) describe six patients with invasive aspergillosis whose only impairment of defence comprised underlying lung disease and corticosteroid treatment. They pointed out two features-firstly, that invasive aspergillosis can also be observed in nonimmunosuppressed patients and, secondly, the role of chronic lung disease as a facilitating factor for its development. We report a patient with idiopathic thrombocytopenic purpura-a condition in which the immunological state of the patient is not impaired-receiving corticosteroids, who developed disseminated aspergillosis without any previous lung disease.

A 63 year old man diagnosed two months earlier as having idiopathic thrombocytopenic purpura presented with fever and progressive dyspnoea. He was having high dose corticosteroids (60-100 mg prednisone daily) and had been diabetic for 10 years.

On admission a chest radiograph showed bilateral pulmonary infiltrates. The white blood cell count was $12.3 \times 10^{9} / 1(90 \%$ neutrophils), haemoglobin $14 \mathrm{~g} / \mathrm{dl}$, and platelet count $65 \times 10^{9} / 1$. He required intubation and mechanical ventilation. Bronchoalveolar lavage showed Pneumocystis carinii. Co-trimoxazole and corticosteroid treatment was started on the fifth day. The patient was seronegative for the human immunodeficiency virus. He improved during the next 10 days but then deteriorated and empirical antibiotic treatment with erythromycin, ceftazidime, amikacin, and amphoteracin B was started. A chest radiograph showed a cavity in the right middle lobe, a right pneumothorax, and pneumomediastinum. Culture of bronchial secretions from a second lavage was positive for Aspergillus fumigatus but no $P$ carinii was identified this time. Despite full dose treatment with amphoteracin $B$, the patient deteriorated and died a week later after invasive aspergillosis was diagnosed and at necropsy $A$ fumigatus was recovered from multiple abcesses in lungs, kidneys, thyroid gland, and heart. The fungus was also found in mitral valve vegetations and in an embolus in the right pulmonary artery.

There are several interesting findings in this patient. Firstly, serious opportunist infections developed without a major cause of immunosuppression (that is, neoplasia or HIV infection), much as in patients 1 and 6 in the series of Dr Palmer and colleagues. Immune disorders caused by diabetes might have played a part along with corticosteroid treatment in the development of this fulminant illness. In recent years the range of underlying diseases associated with aspergillosis has expanded substantially and diabetic patients have developed invasive infections. ${ }^{1}$ Secondly, the infection was extensive, as invasive pulmonary aspergillosis was rapidly followed by fulminant dissemination to the kidneys, heart, thyroid gland, and probably brain. The disseminating pattern differs from that reported by Dr Palmer and colleagues, in which aspergillosis was confined to the lungs. Finally, the presence of $A$ fumigatus endocarditis, an exceptional finding in thrombocytopenic patients, is probably related to platelet malfunction and the low count. $^{2}$

Taken together these confirm that the spectrum of invasive aspergillosis is broader than was originally considered. This infection should be suspected even in patients with minimally compromised immunity. A ORIOL

J M RIBERA

S MART Department of Haematology and Internal Medicine and Intensive Care Unit, Internal Medicine and Intensive Care Unit, 08916 Badalona, Barcelona, Spain

1 Bodey GP, Vartivarian S. Aspergillosis. Eur J Clin Microbiol Infect Dis 1989;8:413-37.

2 Ribera JM, Ferrer O, Blade J, et al. Endocarditis por Aspergillus en un paciente con leucemia aguda linfoblastica en remision. Med Clin aguda linfoblastica en remis
(Barcelona) 1985;81:436-9.

\section{Treatment of pulmonary aspergilloma} with itraconazole

We read with interest the paper of $\mathrm{Dr} \mathrm{J} \mathrm{H}$ Campbell and coworkers (November 1991; 46:839-41). We have had a similar experience with a patient presenting with cough and haemoptysis secondary to aspergilloma (mycetoma), which ws successfully treated with oral itraconazole (100 mg twice daily) given over 10 months. During the course of treatment the size of the mycetoma gradually and progressively decreased and at the completion of treatment could not be identified on a computed tomogram of the chest. Twelve months after cessation of treatment the mycetoma remained unidentifiable.

These observations suggest that aspergilloma can be successfully treated with itraconazole. We agree, however, with Dr Campbell's group that itraconazole should not, at the present time, be recommended for treatment of aspergilloma but should be reserved for cases where treatment is required and a surgical approach is contraindicated. Further experience with this new triazole antifungal agent is necessary, and in the case of its use in the treatment of aspergilloma multicentre study in which substantial numbers of patients could be recruited should be considered.

JONATHAN G W BURDON MAREE BARNES Department of Respiratory Medicine ALAN HARRIS Department of Radiology St Vincent's Hospital, Fitzroy Victoria 3065, Australia

1 Barnes M, Burdon J, Harris A. Itraconazole for pulmonary mycetoma. Med J Aust 1991 154:150.

Symposium on computers in clinical medicine and anaesthesiology

The 13th International Symposium on Computers in Clinical Medicine and Anesthesiology will take place on 11-13 June 1992 in Rotterdam. Details may be obtained from Dr Omar Prakash, Thorax Centre, Dijzig Hospital, Dr Molewaterplein 50, 3015 GD Rotterdam, The Netherlands (tel 31-10-463 5230, fax 31-10-463 5240).

\section{European conference on aerosols}

A European conference on aerosols, organised jointly by the Aerosol Society and its German equivalent, Gesellschaft für Aerosolforschung e V, will be held at Christ Church, Oxford, on 6-11 September 1992 The main topics will be aerosols in the environment, health aspects of aerosols, characterisation of aerosols, industrial aerosols, and fundamental properties of aerosols; the specialist workshops will include one on drug delivery. Further information may be obtained from the conference coordinator, A L Cussens (tel 0275847787 , fax 0275847303 , or Aerosol Society, PO Box 34, Portishead, Bristol BS20 9NR) 